\title{
Barriers and facilitators to the implementation of social robots for older adults and people with dementia: a scoping review
}

Wei Qi Koh ${ }^{1 *}$, Simone Anna Felding ${ }^{2}$, Kübra Beliz Budak², Elaine Toomey ${ }^{3}$ and Dympna Casey ${ }^{1}$

\begin{abstract}
Background: Psychosocial issues, such as social isolation and loneliness among older adults and people with dementia, continue to pose challenges with a rapidly aging population worldwide. Social robots are a rapidly emerging field of technology, developed to help address the psychosocial needs of this population. Although studies have reported positive findings regarding their psychosocial benefits, their implementation in real-world practice remains a challenge. Nevertheless, little is known about the factors affecting their implementation. The purpose of this review is to provide a systematic overview of the barriers and facilitators affecting the implementation of social robots for older adults and people with dementia.

Method: The Arksey and O'Malley approach with methodological enhancement by Levac et al. was used to guide the conduct of this review. Seven electronic databases were searched. In addition, hand searching and backward citation tracing was conducted. Three independent reviewers were involved in the screening and data charting process. Findings were synthesised and categorised into the five domains outlined in the Consolidated Framework of Implementation Research (CFIR).

Results: A total of 53 studies were included in the final review. Most of the included studies were based in participants' homes and in care facilities. Barriers and facilitators were mapped onto 18 constructs in the five domains of the CFIR. The most frequently cited barriers were mapped to the constructs within the domain of "Intervention characteristics", where issues such as the complexity of using the technology and technical obstacles impeded implementation. Most facilitators were mapped onto the domain "Patient needs and resources". Overall, existing research are disproportionately focused on the internal validity (i.e. characteristics) of social robots, and there is significantly less research investigating their external validity, such as organisational or wider contextual factors that can affect their implementation in real-world practice.

Conclusion: This review has identified and synthesised the breadth of evidence on the barriers and facilitators to the implementation of social robots for older adults and people with dementia. Future research should pay more attention to investigating the contextual factors, using an implementation framework, to identify barriers and facilitators to guide the implementation of social robots.
\end{abstract}

Keywords: Social robots, Implementation, Barriers, Facilitators, Scoping review, Consolidated framework for implementation research, Dementia, Older people

\footnotetext{
* Correspondence: weiqi.koh@nuigalway.ie

${ }^{1}$ National University of Ireland Galway, H91 E3YV Galway, Ireland

Full list of author information is available at the end of the article
}

(c) The Author(s). 2021 Open Access This article is licensed under a Creative Commons Attribution 4.0 International License, which permits use, sharing, adaptation, distribution and reproduction in any medium or format, as long as you give appropriate credit to the original author(s) and the source, provide a link to the Creative Commons licence, and indicate if changes were made. The images or other third party material in this article are included in the article's Creative Commons licence, unless indicated otherwise in a credit line to the material. If material is not included in the article's Creative Commons licence and your intended use is not permitted by statutory regulation or exceeds the permitted use, you will need to obtain permission directly from the copyright holder. To view a copy of this licence, visit http://creativecommons.org/licenses/by/4.0/ The Creative Commons Public Domain Dedication waiver (http://creativecommons.org/publicdomain/zero/1.0/) applies to the data made available in this article, unless otherwise stated in a credit line to the data. 


\section{Introduction}

Populations are aging worldwide [1]. It is estimated that $5-8 \%$ of the world's older population live with dementia [2]. Since the prevalence of dementia increases with age [3], it is one of the biggest challenges of a rapidly aging population. Previous research has identified several psychosocial challenges associated with aging and onset of dementia including social isolation, loneliness and a loss of autonomy $[4,5]$. These challenges have continued to place constraints on healthcare costs and caregiving demands [6], which can influence the sustainability of care. Social robots are a rapidly emerging field of technology to facilitate social networks between people, and to interact with people in a meaningful way [7-9]. They provide a multitude of services such as affective therapy, cognitive training and companionship [10] and may be categorised into three operational groups based on their functions: (i) socially assistive robots, (ii) pet robots (or robopets), and (iii) telepresence robots. Socially assistive robots have several functions to assist users with tasks [11], pet robots are intended as viable substitutes to live animals [12] and function as pet therapy to provide physiological and emotional benefits for users [13]. Finally, telepresence robots contain a video conferencing system mounted on a mobile robotic base, and have a primary function to provide social interaction between humans [14]. As such, social robots are considered as a promising technological solution to mitigate some of the challenges associated with rapidly ageing populations by supporting psychosocial needs and assisting with care. A growing body of research focused on developing and evaluating social robots for older people and people with dementia reflects this interest. Their impact and effectiveness have been investigated and synthesized in several reviews [13, 15-17]. Although the overall evidence is not definitive due to insufficient of high-quality studies and smaller sample sizes, synthesised evidence has repeatedly demonstrated strong face validity of their positive impacts in several psychosocial domains, including reduced loneliness, improved social engagement, mood and quality of life $[13,15-17]$. Despite their promise to positively impact the psychosocial health of older adults and people with dementia, their implementation in realpractice remains a challenge $[18,19]$. For example, while $80 \%$ of nursing homes in Denmark have implemented Paro, a pet robot [20], only one dementia care facility has implemented Paro in Ireland [21]. For social robots, the challenges to implementation may be attributed to multi-level factors affecting implementation in actual practice, such as competing demands on the care provider [15], that may not be present or investigated in a research trial due to existence of research supported resources [22]. Additionally, the traditional stepwise approach of research (i.e. investigating implementation only after confirmatory findings of efficacy and effectiveness) has been argued to contribute to the time lag between research discovery and their uptake in real practice [23-25]. To improve the speed of knowledge creation and to improve the clinical relevance of social robots in real-world practice, it is important to pursue knowledge on the implementation of social robots alongside investigation into their effectiveness [26, 27]. Nevertheless, little is known about factors affecting their implementation in practice. A scoping review conducted by Hung et al. [15] found that infection concerns, cost and work load, stigma and ethical issues were key barriers that influenced the adoption of Paro in care settings. In another recent systematic review, Papadopoulos et al. [28] found that facilitators supporting the implementation of socially assistive robots in health and social care settings include the social robots' usability and personalisation, users' enjoyment and familiarity with the technology, while barriers relate to technical issues, limited capabilities of the robots, and users' negative preconceptions. In both two reviews, an implementation framework was not used to guide the search and evidence syntheses, which highlights the possibility that some factors affecting implementation may have been overlooked. Furthermore, there is a variety of terminologies that have been used to describe implementation, which can pose challenges in evidence synthesis [29]. For instance, the term 'implementation' was not used in Papadopoulos et al's [28] search strategy; instead, other terms such as 'service evaluation' and 'acceptability' were used. This issue of terminology variation has also been articulated in another review investigating determinants of implementing e-Health for caregivers of people with dementia, where authors reported that only one out of 46 included articles used the term "implementation" in the title of their publications [30]. There has been no other previous research that has provided a broad overview of the available evidence in this field. Therefore, the objectives of this review were to 1) identify the terminologies that have been used to describe implementation in relation to social robots, and 2) broadly examine existing evidence on barriers and facilitators affecting the implementation of social robots for older adults and people with dementia, and to collate and map the types of available evidence to identify potential research gaps. To address these objectives, a scoping review methodology was identified to be the most appropriate [31].

\section{Conceptual framework}

The Consolidated Framework for Implementation Research (CFIR) was developed by Damschroder and colleagues, based on the integration of 19 different implementation theories, to enable a systematic exploration of multi-level contextual factors that can influence the implementation of an innovation 
or intervention [32]. There are 39 constructs across the five key domains in the CFIR that are reported to influence implementation:

1. Intervention characteristics, which refers to the key attributes of the intervention

2. Outer setting, which refers to external influences on implementation

3. Inner setting, which refers to features of the implementing organisation

4. Characteristics of individuals involved in implementation

5. Implementation process, which refers to the strategies employed in implementation

The CFIR provides a comprehensive approach to the investigation of multi-level barriers and facilitators that can influence implementation. Therefore, employing this framework will enable the identified barriers and facilitators to be presented in a structured and systematic manner. It will also allow findings to be easily compared to other implementation studies to identify research gaps.

\section{Methods}

\section{Protocol and registration}

The Arksey and O'Malley framework [31] for scoping reviews with methodological enhancements by Levac et al. [33], and the Preferred Reporting Items for Systematic Reviews and Meta-analysis Extension for Scoping Reviews (PRSIMA-ScR) [34] (Additional file 1) was used to guide the development, conduct and reporting of this review. The protocol was registered on the Open Science Framework (https://osf.io/2x3y9/), and the methods were described in detail in a published protocol [35].

\section{Stage 1: research question}

The main research question governing this review was: "what is the existing evidence on the barriers and facilitators that affect the implementation of social robots for older people, including people with dementia?"

\section{Stage 2: identifying relevant studies}

A total of seven electronic databases were searched in May 2020, and updated in November 2020: MEDLINE via Ovid, EMBASE, PsycINFO via Ovid, Scopus, Web of Science, Compendex and PubMed. A search strategy was developed in consultation with an expert research librarian using the key terms "older adults", "people with dementia", "social robots" and "implementation". Various terminologies have been used across the literature to describe the concept of implementation. Therefore, we drew on an existing taxonomy of implementation outcomes by Proctor et al. [36] to define the constructs of interest and implementation search terms. They include acceptability, adoption, appropriateness, costs, feasibility, fidelity, penetration and sustainability. A full search strategy for Medline is provided in Additional file 2. We anticipated that the terms "barriers" and "facilitators" may only be discussed in the full-text of articles, potentially described using other terms. As such, these terms were excluded from the search strategy to enable a more thorough search of all research in the field. Consequently, this information was assessed through reading the full texts at a later phase of screening to ensure that no potentially relevant articles were omitted. To identify other potentially relevant studies, the reference list of reviews that were excluded from this study were manually searched [37].

\section{Stage 3: selection of studies}

All search records were imported into Endnote and deduplicated for screening. A two-phased screening process was undertaken by three reviewers (WK, SF, KB). WK screened all articles, while SF and KB each conducted screening of $50 \%$ of all articles independently in each phase. All reviewers met to discuss the results and conflicts after each stage of screening. Firstly, titles and abstracts resulting from the search strategy were selected if they met the following inclusion criteria: (i) used a social robot for more than one session, (ii) involve older adults and/or people with dementia, (iii) contains any terms relevant to any constructs related to implementation, based on Proctor's taxonomy, (iv) published in English language and (v) contains information about barriers and facilitators that influenced implementation. Correspondingly, the exclusion criteria were: (i) non-interventional papers, such as review articles or guidelines, (ii) did not use a social robot, or only used the social robot for a single session, (iii) did not contain any terms relating to implementation and (iv) nonEnglish language publications. Next, full text of relevant papers were then assessed for eligibility for inclusion using the same criteria.

\section{Stage 4: data charting}

A standardised charting form was developed using Microsoft Excel to identify key characteristics of each study, as well as barriers and facilitators to the implementation of social robots. Data that were charted included: authors, publication year, country in which the study was conducted, aims and objectives, study design, study setting, name and type social robot used, intervention characteristics, and barriers and facilitators that influenced implementation. Terms that were used to describe implementation in relation to social robots were charted from the title and abstract of studies. The charting sheet was pre-tested by all reviewers to ensure consistency in data extraction. Three reviewers were 
involved in data charting - WK independently charted all included articles, while SF and KB each charted 50\% of the included articles. All reviewers consulted after the data charting to resolve any inconsistencies.

Stage 5: collating, summarising, and reporting the results WK deductively coded the extracted data by mapping determinants (i.e. barriers or facilitators) onto the 39 constructs in CFIR (Additional file 3). Coded data that were mapped onto each construct were listed, presented in a tabular form and grouped into subcategories. The synthesised results were then organised and presented categorically, based on the five domains in the CFIR. Terms used to describe implementation were mapped onto Proctor's taxonomy of implementation outcomes, and those that are not described in the taxonomy were identified as independent terms. The frequency in which these terms were used were presented.

\section{Results}

The search of databases yielded a total of 1065 publications and an additional 51 from hand searching. After title/abstract screening, 138 articles remained for fulltext screening. A total of 85 publications were excluded after full-text screening (details provided in Additional file 4), and 53 publications that met the eligibility criteria were included in the final review (PRISMA flowchart in Fig. 1). Of these, 18 were published conference papers, and 35 were journal publications.

\section{Study characteristics}

The included publications employed three types of research methods: 15 quantitative $(n=15), 19$ qualitative $(n=19)$ and 19 mixed-method or multi-method $(n=19)$. Studies were conducted in 19 different countries. Most were conducted within 13 countries in Europe $(n=37)$, including Austria, Belgium, Denmark, Finland, France, Greece, Germany, Hungary, Ireland, Italy, the Netherlands, Sweden, and Poland. Others were conducted in Australia $(n=9)$, the United Kingdom $(n=7)$, the United States $(n=5)$, New Zealand $(n=3)$, Japan $(n=2)$ and Mexico $(n=1)$. The majority were conducted in participants' homes $(n=26)$ and long-term care facilities $(n=23)$. Most studies involved older adults $(n=31)$, and people with mild cognitive impairment or dementia $(n=24)$. Some studies also included other stakeholders such as care professionals or management staff $(n=$ $16)$ and family members $(n=12)$. Table 1 shows a summary of the characteristics of included studies.

\section{Social robots and intervention characteristics}

A total of 28 different types of social robots were implemented. This includes 18 types of socially assistive robots $(n=33)$, three types of telepresence robots $(n=8)$ and five types of pet robots $(n=18)$. Paro was the most commonly deployed social robot, and was featured in 11 studies. The intervention duration ranged widely from 2 days to 4 years. Most implemented the social robot over a one-month to three-month period $(n=23)$. In terms of intervention frequency, the majority of studies $(n=$ 19) implemented social robots on a full-time basis, where participants could access the social robot at any time of the day. A summary of this information can be found in Table 2.

Terms used to describe implementation of social robots A total of 13 different terms have been used to describe implementation in relation to social robots (Table 3). Only 15 studies included the term "implement" or "implementation" in their title and/or abstract. Although the term "implementation" was identified in nearly half of the included studies, there appears to be a conceptual overlap on the use of this term. While some authors $(n=8)$ used this term to describe the process of using social robots within a given context [42-44, 48, 49, 68, 82, 83], others $(n=7)$ used it to describe the execution of technical or systems of the social robot $[47,56-58,81,85,90]$. Out of the eight constructs in Proctor's taxonomy, we identified terms that could be mapped onto five. Overall, "acceptability" or "acceptance" were most frequently used terms $(n=25)$. Other terms that were used included use, usefulness, integration, usability and deployment.

\section{Barriers and facilitators to implementation}

A summary of barriers and facilitators coded to the CFIR, excluding constructs with no supporting data, are presented in Table 4 . Overall, the barriers and facilitators were mapped onto 18 constructs across all five domains. There was no data that could be mapped onto the 21 other CFIR constructs.

\section{Domain 1: innovation characteristics Relative advantage}

Telepresence robots were considered to be more disadvantageous than using the telephone or skype as they were more expensive [70] and had less audibility to cater to those with a hearing impairment $[38,75,76]$. Relative advantages included an increased sense of presence due to their video element [38, 70, 74-76] and mobility aspect [74]. They were also reported to be more conducive for use with people with dementia [70, 74]. Pet robots were compared to live animals, where their maintenance-free nature was seen as an advantage [60, 72]. Socially assistive robots were perceived to be more beneficial than a tablet solution due to their proactivity [56], and potential economic profitability as compared to having human staff [59]. 


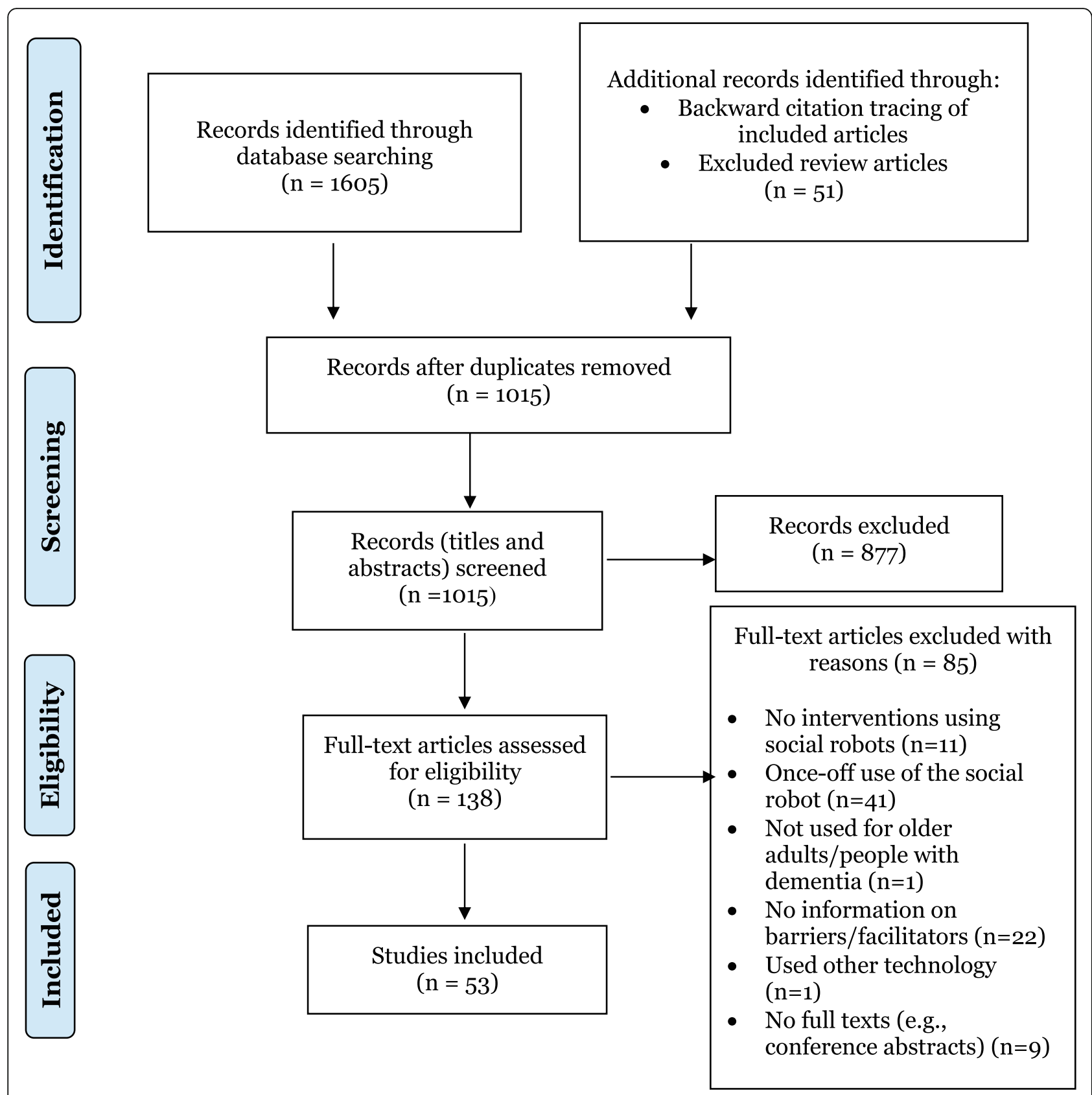

Fig. 1 PRISMA Flow Diagram

\section{Adaptability}

The inability to adapt the functions of social robots to cater to participants' preferences and abilities impeded their use. This included the inability to adjust vocalisations [83], personalise functions [45], and customise user interfaces or modes of robot interaction [41, 46, 53, 63]. Other barriers relate to issues of physical inaccessibility [41, 47, 59, 68, 74, 77, 78, 81, 84]. Correspondingly, facilitators included the physical accessibility [41, 74] and customisability of the robots' interactivity or functions $[47,64]$.

\section{Complexity}

The complexity of operating social robots primarily related to the use of socially assistive robots, which included complicated pre-programmed instructions [39, 46] and functions [39, 41, 75-77, 83, 85, 88], or difficulty composing or programming activities [61]. For telepresence robots, navigation difficulties occurred during remote driving [69, 75, 76]. For some participants, particularly people with dementia, the multiple modes of visual, auditory and tactile interaction with social robots were confusing and challenging $[41,67,75,76]$. 
Table 1 Characteristics of included studies

\begin{tabular}{|c|c|c|c|c|c|c|}
\hline Author & Country & $\begin{array}{l}\text { Publication } \\
\text { type }\end{array}$ & Methodology & Study design & Study participants & Study setting \\
\hline $\begin{array}{l}\text { Aaltonen et al., } \\
2017 \text { [38] }\end{array}$ & Finland & $\begin{array}{l}\text { Conference } \\
\text { paper }\end{array}$ & Qualitative & $\begin{array}{l}\text { Qualitative interviews, } \\
\text { observations }\end{array}$ & $\begin{array}{l}\text { Older person, care staff, family } \\
\text { members }\end{array}$ & Participants' homes \\
\hline $\begin{array}{l}\text { Bajones et al., } \\
2018 \text { [39] }\end{array}$ & $\begin{array}{l}\text { Austria, } \\
\text { Greece, } \\
\text { Sweden }\end{array}$ & $\begin{array}{l}\text { Journal } \\
\text { paper }\end{array}$ & Multi-method & Field trial & $\begin{array}{l}\text { Older people (living alone, } \\
\text { fallen in the last } 2 \text { years, and } \\
\text { impairments in mobility, }\end{array}$ & Participants' homes \\
\hline $\begin{array}{l}\text { Bajones et al., } \\
2019 \text { [40] }\end{array}$ & $\begin{array}{l}\text { Austria, } \\
\text { Greece, } \\
\text { Sweden }\end{array}$ & $\begin{array}{l}\text { Journal } \\
\text { paper }\end{array}$ & Multi-method & Field trial & Older people (living alone) & Participants' homes \\
\hline $\begin{array}{l}\text { Barrett et al., } \\
2019 \text { [41] }\end{array}$ & Ireland & $\begin{array}{l}\text { Journal } \\
\text { paper }\end{array}$ & Quantitative & $\begin{array}{l}\text { Single group, pre-post } \\
\text { pilot study }\end{array}$ & People with dementia & Nursing home \\
\hline $\begin{array}{l}\text { Bemelmens et al., } \\
2016 \text { [42] }\end{array}$ & Netherlands & $\begin{array}{l}\text { Journal } \\
\text { paper }\end{array}$ & Multi-method & Feasibility study & $\begin{array}{l}\text { People with dementia, care } \\
\text { staff, family members }\end{array}$ & $\begin{array}{l}\text { Care institution for } \\
\text { psychogeriatric care }\end{array}$ \\
\hline Blond, 2019 [43] & $\begin{array}{l}\text { Denmark, } \\
\text { Finland }\end{array}$ & $\begin{array}{l}\text { Journal } \\
\text { paper }\end{array}$ & Qualitative & Ethnographic study & $\begin{array}{l}\text { Older adults, care staff, } \\
\text { management staff }\end{array}$ & Elderly care center \\
\hline $\begin{array}{l}\text { Bradwell et al., } \\
2020 \text { [44] }\end{array}$ & UK & $\begin{array}{l}\text { Conference } \\
\text { paper }\end{array}$ & Qualitative & Longitudinal study & Older people & Supported living facility \\
\hline $\begin{array}{l}\text { Broadbent et al., } \\
2014 \text { [45] }\end{array}$ & $\begin{array}{l}\text { New } \\
\text { Zealand }\end{array}$ & $\begin{array}{l}\text { Conference } \\
\text { paper }\end{array}$ & Quantitative & $\begin{array}{l}\text { Repeated measures } \\
\text { randomised cross-over trial }\end{array}$ & Older people & Participants' homes \\
\hline $\begin{array}{l}\text { Caleb-Solly et al., } \\
2018 \text { [46] }\end{array}$ & $\begin{array}{l}\text { UK, } \\
\text { Netherlands }\end{array}$ & $\begin{array}{l}\text { Journal } \\
\text { paper }\end{array}$ & Quantitative & $\begin{array}{l}\text { Usability and user } \\
\text { experience evaluation }\end{array}$ & Older people & $\begin{array}{l}\text { Assisted living studio, } \\
\text { residential care, and } \\
\text { participants' homes }\end{array}$ \\
\hline $\begin{array}{l}\text { Carros et al., } 2020 \\
\text { [47] }\end{array}$ & Germany & $\begin{array}{l}\text { Conference } \\
\text { paper }\end{array}$ & Qualitative & Pre and post interviews & $\begin{array}{l}\text { Older people, caregivers and } \\
\text { manager }\end{array}$ & Care home \\
\hline $\begin{array}{l}\text { Chang et al., } \\
2013 \text { [48] }\end{array}$ & USA & $\begin{array}{l}\text { Conference } \\
\text { paper }\end{array}$ & Multi-method & $\begin{array}{l}\text { Observations and } \\
\text { interview }\end{array}$ & Older people, care staff & $\begin{array}{l}\text { Retirement community } \\
\text { (long- and short-term } \\
\text { care) }\end{array}$ \\
\hline $\begin{array}{l}\text { Chang et al., } \\
2015 \text { [49] }\end{array}$ & USA & $\begin{array}{l}\text { Conference } \\
\text { paper }\end{array}$ & Multi-method & Field study & $\begin{array}{l}\text { Older people (majority had } \\
\text { dementia), staff, visitors }\end{array}$ & Nursing home \\
\hline $\begin{array}{l}\text { Cruz-Sandoval } \\
\text { et al., } 2018 \text { [50] }\end{array}$ & Mexico & $\begin{array}{l}\text { Conference } \\
\text { paper }\end{array}$ & Quantitative & Observational & Older people with dementia & Geriatric residence \\
\hline $\begin{array}{l}\text { de Graaf et al., } \\
2015 \text { [51] }\end{array}$ & UK & $\begin{array}{l}\text { Journal } \\
\text { paper }\end{array}$ & Qualitative & $\begin{array}{l}\text { Exploratory in-depth study } \\
\text { using video recording and } \\
\text { interviews }\end{array}$ & Older people & Participants' homes \\
\hline $\begin{array}{l}\text { Demange et al., } \\
2018 \text { [52] }\end{array}$ & France & $\begin{array}{l}\text { Journal } \\
\text { paper }\end{array}$ & Quantitative & $\begin{array}{l}\text { Quasi-experimental (pre- } \\
\text { post) }\end{array}$ & Older people with dementia & Hospital \\
\hline $\begin{array}{l}\text { D'Onofrio et al., } \\
2019 \text { [53] }\end{array}$ & Italy & $\begin{array}{l}\text { Conference } \\
\text { paper }\end{array}$ & Quantitative & Pre-post & Older people with dementia & Hospital \\
\hline $\begin{array}{l}\text { D'Onofrio et al., } \\
2019 \text { [53] }\end{array}$ & $\begin{array}{l}\text { Italy, Ireland } \\
\text { and UK }\end{array}$ & $\begin{array}{l}\text { Journal } \\
\text { paper }\end{array}$ & Quantitative & Pre-post & People with dementia & $\begin{array}{l}\text { Community setting, } \\
\text { nursing home and } \\
\text { hospital }\end{array}$ \\
\hline $\begin{array}{l}\text { Fattal et al., } 2020 \\
\text { [54] }\end{array}$ & France & $\begin{array}{l}\text { Journal } \\
\text { paper }\end{array}$ & Quantitative & Pre-post & Older people & Hospital \\
\hline $\begin{array}{l}\text { Fiorini et al., } 2020 \\
\text { [55] }\end{array}$ & Italy & $\begin{array}{l}\text { Conference } \\
\text { paper }\end{array}$ & Quantitative & Pre-post & Older people & Participants' homes \\
\hline $\begin{array}{l}\text { Gross et al., } 2012 \\
\text { [56] }\end{array}$ & $\begin{array}{l}\text { Netherlands } \\
\text { Belgium }\end{array}$ & $\begin{array}{l}\text { Conference } \\
\text { paper }\end{array}$ & Qualitative & Field trial & $\begin{array}{l}\text { Older people with mild } \\
\text { cognitive impairment and } \\
\text { their partner }\end{array}$ & $\begin{array}{l}\text { Smart home (Test } \\
\text { home) }\end{array}$ \\
\hline $\begin{array}{l}\text { Gross et al., } 2015 \\
\text { [57] }\end{array}$ & Germany & $\begin{array}{l}\text { Conference } \\
\text { paper }\end{array}$ & Multi-method & Case study & Older people & Participants' homes \\
\hline $\begin{array}{l}\text { Gross et al., } 2019 \\
\text { [58] }\end{array}$ & Germany & $\begin{array}{l}\text { Conference } \\
\text { paper }\end{array}$ & Multi-method & Case study & Older people & Participants' homes \\
\hline $\begin{array}{l}\text { Hebesberger } \\
\text { et al., } 2017 \text { [59] }\end{array}$ & Austria & $\begin{array}{l}\text { Journal } \\
\text { paper }\end{array}$ & $\begin{array}{l}\text { Mixed } \\
\text { method }\end{array}$ & $\begin{array}{l}\text { Concurrent multistrand } \\
\text { research design }\end{array}$ & $\begin{array}{l}\text { Older people with dementia, } \\
\text { care staff and management } \\
\text { staff }\end{array}$ & Hospital \\
\hline
\end{tabular}


Table 1 Characteristics of included studies (Continued)

\begin{tabular}{|c|c|c|c|c|c|c|}
\hline Author & Country & $\begin{array}{l}\text { Publication } \\
\text { type }\end{array}$ & Methodology & Study design & Study participants & Study setting \\
\hline $\begin{array}{l}\text { Hudson et al., } \\
2020 \text { [60] }\end{array}$ & USA & $\begin{array}{l}\text { Journal } \\
\text { paper }\end{array}$ & Qualitative & Descriptive qualitative & Older people & Participants' homes \\
\hline $\begin{array}{l}\text { Huisman and } \\
\text { Kort, } 2019 \text { [61] }\end{array}$ & Netherlands & $\begin{array}{l}\text { Journal } \\
\text { paper }\end{array}$ & $\begin{array}{l}\text { Mixed } \\
\text { method }\end{array}$ & Evaluation study & $\begin{array}{l}\text { Older adults, care staff and } \\
\text { board members }\end{array}$ & Geriatric care facilities \\
\hline $\begin{array}{l}\text { Kelly et al., } 2020 \\
\text { [62] }\end{array}$ & USA & $\begin{array}{l}\text { Journal } \\
\text { paper }\end{array}$ & Quantitative & Feasibility study & Older people with dementia & Hospital (acute care) \\
\hline $\begin{array}{l}\text { Khosla et al., } \\
2017 \text { [63] }\end{array}$ & Australia & $\begin{array}{l}\text { Journal } \\
\text { paper }\end{array}$ & Quantitative & Cross-sectional & Older people with dementia & $\begin{array}{l}\text { Residential aged care } \\
\text { facilities }\end{array}$ \\
\hline $\begin{array}{l}\text { Khosla et al., } \\
2019 \\
\text { (Australia) [64] }\end{array}$ & Australia & $\begin{array}{l}\text { Journal } \\
\text { paper }\end{array}$ & $\begin{array}{l}\text { Mixed } \\
\text { method }\end{array}$ & Observational & $\begin{array}{l}\text { People with dementia, family } \\
\text { members }\end{array}$ & Participants' homes \\
\hline $\begin{array}{l}\text { Klamer et al., } \\
2010 \text { [65] }\end{array}$ & UK & $\begin{array}{l}\text { Conference } \\
\text { paper }\end{array}$ & Qualitative & Case study & Older people & Participants' homes \\
\hline $\begin{array}{l}\text { Kolstad et al., } \\
2020[66]\end{array}$ & Japan & $\begin{array}{l}\text { Journal } \\
\text { paper }\end{array}$ & Qualitative & Semi structured interviews & $\begin{array}{l}\text { Older people, nursing staff } \\
\text { and site managers }\end{array}$ & $\begin{array}{l}\text { Two nursing homes } \\
\text { and one elderly day } \\
\text { care centre }\end{array}$ \\
\hline $\begin{array}{l}\text { Kouroupetroglou } \\
\text { et al., } 2017 \text { [67] }\end{array}$ & Italy, Ireland & $\begin{array}{l}\text { Conference } \\
\text { paper }\end{array}$ & Quantitative & Questionnaire & People with dementia & $\begin{array}{l}\text { Hospital and nursing } \\
\text { home }\end{array}$ \\
\hline $\begin{array}{l}\text { Melkas et al., } \\
2020 \text { [68] }\end{array}$ & Finland & $\begin{array}{l}\text { Journal } \\
\text { paper }\end{array}$ & Qualitative & Field study & Older people, care staff & $\begin{array}{l}2 \text { care homes and a } \\
\text { geriatric rehabilitation } \\
\text { hospital }\end{array}$ \\
\hline $\begin{array}{l}\text { Moyle et al., } 2013 \\
\text { [69] }\end{array}$ & Australia & $\begin{array}{l}\text { Conference } \\
\text { paper }\end{array}$ & Qualitative & Case study & Older people with dementia & Nursing home \\
\hline $\begin{array}{l}\text { Moyle et al., } 2014 \\
\text { [70] }\end{array}$ & Australia & $\begin{array}{l}\text { Journal } \\
\text { paper }\end{array}$ & $\begin{array}{l}\text { Mixed } \\
\text { method }\end{array}$ & $\begin{array}{l}\text { Semi structured interviews } \\
\text { and observational data }\end{array}$ & $\begin{array}{l}\text { Older people with dementia, } \\
\text { care staff, family members }\end{array}$ & $\begin{array}{l}\text { Long term care } \\
\text { facilities }\end{array}$ \\
\hline $\begin{array}{l}\text { Moyle et al., } 2016 \\
\text { [71] }\end{array}$ & Australia & $\begin{array}{l}\text { Journal } \\
\text { paper }\end{array}$ & Qualitative & Case study & Older people with dementia & Nursing home \\
\hline $\begin{array}{l}\text { Moyle et al., } 2019 \\
\text { [72] }\end{array}$ & Australia & $\begin{array}{l}\text { Journal } \\
\text { paper }\end{array}$ & Qualitative & Descriptive qualitative & $\begin{array}{l}\text { Family members of older } \\
\text { people who live in residential } \\
\text { care }\end{array}$ & $\begin{array}{l}\text { Residential care } \\
\text { facilities }\end{array}$ \\
\hline $\begin{array}{l}\text { Moyle et al., } 2019 \\
\text { [73] }\end{array}$ & Australia & $\begin{array}{l}\text { Journal } \\
\text { paper }\end{array}$ & Qualitative & Descriptive qualitative & Older people with dementia & Long term care facility \\
\hline $\begin{array}{l}\text { Moyle et al., } 2019 \\
\text { [74] }\end{array}$ & Australia & $\begin{array}{l}\text { Journal } \\
\text { paper }\end{array}$ & Qualitative & Descriptive qualitative & $\begin{array}{l}\text { People with dementia, family } \\
\text { members }\end{array}$ & Long term care facility \\
\hline $\begin{array}{l}\text { Niemala et al., } \\
2017 \text { [75] }\end{array}$ & Finland & $\begin{array}{l}\text { Conference } \\
\text { paper }\end{array}$ & Qualitative & $\begin{array}{l}\text { Pre-post interviews, user } \\
\text { observations, logged use } \\
\text { of robot, videotaping }\end{array}$ & Older people & $\begin{array}{l}\text { Long term residential } \\
\text { home }\end{array}$ \\
\hline $\begin{array}{l}\text { Niemala et al., } \\
2019 \text { [76] }\end{array}$ & Finland & $\begin{array}{l}\text { Journal } \\
\text { paper }\end{array}$ & Multi-method & Field trial & $\begin{array}{l}\text { Older people, care staff, family } \\
\text { members }\end{array}$ & $\begin{array}{l}\text { Residential care } \\
\text { facilities }\end{array}$ \\
\hline $\begin{array}{l}\text { Orejana et al., } \\
2015[77]\end{array}$ & $\begin{array}{l}\text { New } \\
\text { Zealand }\end{array}$ & $\begin{array}{l}\text { Conference } \\
\text { paper }\end{array}$ & Multi-method & Case study & Older people & Participants' homes \\
\hline $\begin{array}{l}\text { Peri et al., } 2016 \\
\text { [78] }\end{array}$ & $\begin{array}{l}\text { New } \\
\text { Zealand }\end{array}$ & $\begin{array}{l}\text { Journal } \\
\text { paper }\end{array}$ & Quantitative & $\begin{array}{l}\text { Controlled non- } \\
\text { randomised comparison } \\
\text { study (Observational) }\end{array}$ & $\begin{array}{l}\text { Older people, care staff, } \\
\text { visitors }\end{array}$ & $\begin{array}{l}\text { Retirement complex } \\
\text { (Residential care ward) }\end{array}$ \\
\hline $\begin{array}{l}\text { Piasek and } \\
\text { Wieczororwska- } \\
\text { Tobis, } 2018 \text { [79] }\end{array}$ & Poland & $\begin{array}{l}\text { Journal } \\
\text { paper }\end{array}$ & Quantitative & Pre-post & $\begin{array}{l}\text { Older people with mild } \\
\text { cognitive impairment, family } \\
\text { members }\end{array}$ & $\begin{array}{l}\text { Laboratory setting and } \\
\text { participants' homes }\end{array}$ \\
\hline $\begin{array}{l}\text { Pike et al., } 2020 \\
\text { [80] }\end{array}$ & UK & $\begin{array}{l}\text { Journal } \\
\text { paper }\end{array}$ & Qualitative & Multiple case study & $\begin{array}{l}\text { Older people with dementia, } \\
\text { family members }\end{array}$ & Participants' homes \\
\hline $\begin{array}{l}\text { Portugal et al., } \\
2019 \text { [81] }\end{array}$ & Netherlands & $\begin{array}{l}\text { Journal } \\
\text { paper }\end{array}$ & Multi-method & $\begin{array}{l}\text { Observation and post- } \\
\text { questionnaire }\end{array}$ & $\begin{array}{l}\text { Older people, care staff, } \\
\text { visitors }\end{array}$ & Care center \\
\hline $\begin{array}{l}\text { Pu et al., } 2020 \\
\text { [82] }\end{array}$ & Australia & $\begin{array}{l}\text { Journal } \\
\text { paper }\end{array}$ & Qualitative & Descriptive qualitative & Older people with dementia & $\begin{array}{l}\text { Residential aged care } \\
\text { facility }\end{array}$ \\
\hline Randall et al., & USA & Journal & Multi-method & Pre-post focus groups, & Older people & Participants' homes \\
\hline
\end{tabular}


Table 1 Characteristics of included studies (Continued)

\begin{tabular}{|c|c|c|c|c|c|c|}
\hline Author & Country & $\begin{array}{l}\text { Publication } \\
\text { type }\end{array}$ & Methodology & Study design & Study participants & Study setting \\
\hline 2019 [83] & & paper & & survey & & \\
\hline $\begin{array}{l}\text { Sabelli et al., } \\
2011 \text { [84] }\end{array}$ & Japan & $\begin{array}{l}\text { Conference } \\
\text { paper }\end{array}$ & Qualitative & Ethnographic study & Older people, care staff & Elderly care center \\
\hline $\begin{array}{l}\text { Schroeter et al., } \\
2013 \text { [85] }\end{array}$ & $\begin{array}{l}\text { Netherlands } \\
\text { Belgium }\end{array}$ & $\begin{array}{l}\text { Journal } \\
\text { paper }\end{array}$ & Multi-method & $\begin{array}{l}\text { Semi-structured interviews, } \\
\text { observation, diary, } \\
\text { questionnaire }\end{array}$ & $\begin{array}{l}\text { Older people with mild } \\
\text { cognitive impairment and } \\
\text { their partner }\end{array}$ & $\begin{array}{l}\text { Smart home (Test } \\
\text { home) }\end{array}$ \\
\hline $\begin{array}{l}\text { Torta et al., } 2014 \\
\text { [86] }\end{array}$ & Austria & $\begin{array}{l}\text { Journal } \\
\text { paper }\end{array}$ & Multi-method & $\begin{array}{l}\text { Questionnaire and semi- } \\
\text { structured interviews }\end{array}$ & Older people & $\begin{array}{l}\text { Test setting (In a } \\
\text { Senior centre) }\end{array}$ \\
\hline $\begin{array}{l}\text { van Maris et al., } \\
2020 \text { [87] }\end{array}$ & UK & $\begin{array}{l}\text { Journal } \\
\text { paper }\end{array}$ & Multi-method & $\begin{array}{l}\text { Questionnaire and } \\
\text { interviews }\end{array}$ & Older people & Retirement villages \\
\hline $\begin{array}{l}\text { Wu et al., } 2014 \\
\text { [88] }\end{array}$ & France & $\begin{array}{l}\text { Journal } \\
\text { paper }\end{array}$ & Multi-method & $\begin{array}{l}\text { Questionnaire and semi- } \\
\text { structured interviews }\end{array}$ & $\begin{array}{l}\text { Older people (cognitively } \\
\text { healthy and those with mild } \\
\text { cognitive impairment) }\end{array}$ & $\begin{array}{l}\text { Test setting (In the } \\
\text { Gerontechnology living } \\
\text { lab in a hospital) }\end{array}$ \\
\hline $\begin{array}{l}\text { Zsiga et al., } 2018 \\
\text { [89] }\end{array}$ & Hungary & $\begin{array}{l}\text { Journal } \\
\text { paper }\end{array}$ & Quantitative & Field test & Older people & Participants' homes \\
\hline
\end{tabular}

Facilitators relating to their ease of use were reported in 14 studies [39-41, 52, 57, 59-61, 65, 68, 74, 76, 81, 88], of which some attributed this to the involvement of users in the design process [41] and prolonged technology use [86].

\section{Design quality and packaging}

Technical issues were widely reported as barriers, particularly in relation to socially assistive robots. These included audio and speech issues [39-41, 43, 46, 47, 53, $63,71,74-76,81,84,86,88,89]$, hardware problems [43, 58, 70], overheating [40, 69, 70], unreliability of functions $[39,40,43,45,46,58,59,65,71,81,85,89$, $90]$, unclear or unpredictable actions [39, 40, 43, 51] and other technical issues [43, 47, 54, 61, 77]. The frequent need to recharge batteries was also cited as a barrier [83]. Next, barriers relating to their physical attributes, such as weight $[16,72]$, size $[45,68]$ unpleasant vocalisations $[16,67,83]$ and unsatisfactory levels of interactivity $[16,83]$, were raised. Finally, unfamiliar designs [72, 83] and the "machine-like" [71, 81] or "toy-like" [71] appearances of social robots were also cited as issues. Facilitators were related to overall acceptable or pleasant appearances and design $[41,45,48,49,54,63,64,67$, $68,82,86,88]$. Other facilitators included the interactivity and proactivity of social robots $[40,41,57,58,77,84$, $85]$, and their overall robustness [44, 57, 89].

\section{Cost}

Multiple stakeholders raised concerns about high acquisition costs $[44,57,69,72,83,88]$, and maintenance costs of social robots, especially when used in rural areas or out of their country of manufacture $[69,77]$.

\section{Domain 2: outer setting \\ Patient needs and resources}

The demographics of participants influenced their needs. Older people who were less familiar with technology were more hesitant to use social robots [51, 74, 88]. People with dementia, especially those with more cognitive impairment, required more ongoing support [41, 48, 49, 53, 67, 74, 88]. Correspondingly, familiarisation and support to use the technology was perceived to be a necessary facilitator $[47,57,79,88]$. Next, the inability of social robots to meet participants' needs also impeded their use. Older adults who were living at home and were independent in managing daily tasks felt that the technology was unnecessary $[60,77,88]$, had limited usefulness [40, 41, 45, 51, 57, 65, 83, 87], and had doubts about their benefits with sustained use [57, 86, 88]. Issues that were raised by both older adults and people with dementia include privacy concerns $[45,46,51,57$, $83,88]$, negative affect which stemmed from technical issues $[40,47,53,59,64,65,71,88]$, and negative perceptions or stigma $[40,44,51,52,54,55,62,71,80,81,88]$. Correspondingly, when functions of the robots aligned with participants' needs and were perceived to be relevant, their use was facilitated. The needs that these robots fulfilled included emotional support [41, 52, 57, 58, 60, 82-85], companionship [44, 45, 60, 77, 82, 83], perceived improvements to daily life $[40,58,63,81]$, entertainment $[41,45,50,63,64]$, reminiscence $[41,45,71]$ and non-intrusive reminders $[54,58,64]$. Phased introduction and training [46] and familiarisation also facilitated a greater acceptance of [70] and adaptation to the technology [46, 47, 51].

\section{External policy and incentive}

Only two studies $(n=2)$ reported on external policy as a facilitator, where care professionals perceived that use of 
Table 2 Social robot(s) and intervention characteristics

\begin{tabular}{|c|c|}
\hline & No. of studies (n) \\
\hline \multicolumn{2}{|l|}{ Social robots used } \\
\hline Pet robots & 18 \\
\hline Paro & $11[42,48,49,52,62,66,69,72,82,83]$ \\
\hline CuDDler & $1[71]$ \\
\hline Qooboo & $1[66]$ \\
\hline Joy for all cat & $3[44,60,80]$ \\
\hline Joy for all dog & $2[44,60]$ \\
\hline Telepresence robots & 8 \\
\hline VGo & $1[74]$ \\
\hline Giraff & $3[69,70,74]$ \\
\hline Double & $4[38,55,75,76]$ \\
\hline Socially assistive robots & 33 \\
\hline Betty / Matilda & $2[63,64]$ \\
\hline Cafero & $2[45,78]$ \\
\hline CompanionAble robot & $2[56,85]$ \\
\hline Eva & $1[50]$ \\
\hline Guide & $1[78]$ \\
\hline Hobbit PT2 & $1[39,40]$ \\
\hline iRobiQ & $2[45,77]$ \\
\hline Kompai mobile robot & $3[46,88,89]$ \\
\hline MARIO & $4[41,53,67,90]$ \\
\hline MAX (SCITOS G3) & $1[57]$ \\
\hline Nao / Zora & $3[61,68,86]$ \\
\hline Pepper & $4[47,54,66,87]$ \\
\hline Robovie 2 & $1[84]$ \\
\hline Silbot-2 & $1[43]$ \\
\hline STRANDS robot & $1[59]$ \\
\hline SYMPARNTER & $1[58]$ \\
\hline Tiago & $1[79]$ \\
\hline Violet's Nabaztag & $2[51,65]$ \\
\hline \multicolumn{2}{|l|}{ Study Duration } \\
\hline Less than 1 week & $6[53,56,57,62,81,85]$ \\
\hline One to four weeks & $\begin{array}{l}14[39-42,52,54,58,59,65,67,68,82,87, \\
88]\end{array}$ \\
\hline More than four to 12 weeks & $\begin{array}{l}23[38,45-51,60,64,70-76,78-80,82,86 \text {, } \\
89]\end{array}$ \\
\hline More than 12 weeks & $6[43,61,63,77,84,91]$ \\
\hline No clear information & $4[53,55,66,69]$ \\
\hline \multicolumn{2}{|l|}{ Intervention Frequency } \\
\hline Full-time (or full day) & $\begin{array}{l}20[38-40,45,51,55-60,65,77,79,81,82, \\
85,89]\end{array}$ \\
\hline $\begin{array}{l}\text { Weekly intervention (ranging } \\
\text { from } 1 \text { to } 5 \text { times weekly) }\end{array}$ & $\begin{array}{l}18[41,42,47-50,52-54,61,64,67,71-73 \\
75,82-88]\end{array}$ \\
\hline Others & $2[86,91]$ \\
\hline No clear information & $\begin{array}{l}13[43,46,53,62,63,66,68-70,74,76,80, \\
84]\end{array}$ \\
\hline
\end{tabular}

Table 3 Terms used to describe implementation

\begin{tabular}{ll}
\hline Terms used & No of studies $\mathbf{( n )}$ \\
\hline $\begin{array}{l}\text { Proctor's taxonomy } \\
\text { acceptability, }\end{array}$ & $25[40,41,45,46,50-56,58,59,62,63,65,67,77$, \\
acceptance & $79,80,86-90]$ \\
adoption, adopt & $6[53,60,75,76,84,88]$ \\
feasibility & $8[38,42,45,54,62,70,71,74]$ \\
sustainability & $1[63]$ \\
$\begin{array}{l}\text { cost } \\
\text { penetration }\end{array}$ & $1[72]$ \\
fidelity & no data \\
$\begin{array}{l}\text { appropriateness } \\
\text { Other terms }\end{array}$ & no data \\
$\begin{array}{l}\text { implementation, } \\
\text { implement }\end{array}$ & $15[42-44,47-49,56-58,68,81-83,85,90]$ \\
$\begin{array}{l}\text { use, usage } \\
\text { usefulness, useful }\end{array}$ & $8[45,49,53,54,63,83,88,89]$ \\
integrate, & $5[54,59,60,66,68]$ \\
integration & $71-73,75,76,78-80,82,83,85,88,90]$ \\
$\begin{array}{l}\text { usability } \\
\text { deploy, } \\
\text { deployment }\end{array}$ & $4[40,46,54,55]$ \\
utilisation, utilise & $2[66,78]$ \\
employ & $1[40]$ \\
\hline &
\end{tabular}

the technology aligned their care work with the wider national care policy $[75,76]$.

\section{Domain 3: inner setting}

\section{Compatibility}

In care facilities, barriers included institutional regulations which limited the mobility of social robots due to issues of privacy $[38,75]$, safety and space allocation [84]. The unexpected appearances of the robot confused some residents [59], and background noises also influenced participants' interaction with the technology [41, 53, 75]. Next, challenges integrating social robots into work process included concerns about potential misuse of the technology $[38,75,76]$, lack of support from coworkers [61], uncertainty on how to delineate a professional boundary $[38,75,76]$, ethical $[42,68,71,73]$, and hygiene concerns [42, 44, 72, 73]. Correspondingly, they were compatible with work processes when their use supported the work of care workers [47, 59, 68, 76, 84], could be integrated into daily care routine [42, 47, 49, $75,84]$. For studies conducted in participants' homes, incompatibility occurred when social robots interfered with daily routine $[47,51]$, or when environment inaccessibility impeded the robots' mobility $[40,51,58]$. Facilitators included an integrated routine of use [51, 60, 65], and environment accessibility [40, 41]. 


\section{Relative priority}

Barriers relating to relative priority were reported in three studies $(n=3)$, where care professionals felt that social robots caused additional work, and that existing work took precedence $[66,68,75]$. Their use also led to workplace tension, where those who did not prioritise use of the technology dissented those who used it [68].

\section{Leadership engagement}

Only one study $(n=1)$ reported on leadership engagement as a facilitator, where organisational leaders demonstrated active involvement and commitment towards implementation effort. Support services and meetings were planned for care professionals to exchange knowledge and experiences [61].

\section{Available resources}

More resource-related barriers than facilitators were identified. In care facilities, barriers included poor network connectivity [38, 39, 61, 68, 70, 74-76, 81], and lack of manpower, time or training [42, 66, 68-70]. Only one study reported on facilitators, where the network infrastructure was boosted, and time and support were provided to support use of the technology [61]. For studies that were conducted in participants' homes, or involved family members who lived at home, resource barriers include a lack of Wi-Fi infrastructure [55] and computer incompatibility [74] to connect with the robot at the care facility.

\section{Access to knowledge and information}

Access to technical support was reported as a barrier for participants who lived in rural areas [77]. Three studies reported access to knowledge and information within care facilities through a dedicated helpdesk [61], a manual and individualised interventions instructions [42, 43], which supported implementation.

\section{Domain 4: characteristics of individuals Knowledge and beliefs}

Some care workers and family members were ambivalent or had negative attitudes towards social robots [42, 47, $59,66,68,72,74,81]$, hesitated their use for fear of damaging them $[59,77]$, and had concerns about privacy $[38,75,76]$ and job replacement by robots $[47,59]$. While some negative perceptions persisted after experiencing their use, due to technical challenges or perceived lack of usefulness $[59,61,74,75]$, other attitudes evolved positively after witnessing their positive impacts [42, 44, $47,49,56,66,68-70,72,74,75,80,81]$, and having a renewed understanding that robots cannot replace their jobs [47]. As such, they were motivated and willing to support robot interactions [42, 61, 84]. Perceptions at the managerial level were only reported in one study $(n=1)$, which reported positive views that the technology aligned with the organisation's vision [61].

\section{Self efficacy}

Only one study $(n=1)$ reported that care workers felt unequipped to compose group activities using social robots. Nevertheless, they gained experience to work around the capabilities of the technology over time [61].

\section{Planning}

In one study $(n=1)$, the plan to assign a social robot with a clear role to make it more approachable facilitated the implementation process [84].

\section{Engaging}

The public exposure of social robots facilitated engagement by multiple stakeholders [59], who developed positive perceptions of the value of the technology from observing robot interactions $[49,70]$.

\section{Key stakeholders}

Negative attitudes of care professionals was reported as a key barrier to implementation [69], while staff enthusiasm was facilitated their use [66]. Only one study $(n=1)$ reported active involvement of care professionals in the implementation process, which facilitated their proactivity and enthusiasm [84]. Staff-mediated robot interactions, such as using active strategies to mediate the limitations of robot interactions [43, 47, 49, 50,67] and changing composition of group sessions [48] led to more successful robot interactions.

\section{External change agents}

Eight studies $(n=8)$ identified family members, researchers and robot developers to be external change agents, who facilitated the implementation process by supporting participants' interactions with social robots $[40,41,49,74,76]$ and providing technical support [39, $43,77]$. However, the ethical challenge of lack of sustainability of social robot intervention after the end of the study was reported in one study [47].

\section{Discussion}

This review synthesises available evidence on the barriers and facilitators to the implementation of social robots for older people and people with dementia. Most included studies were conducted in long term care facilities and in participants' homes, and the majority used socially assistive robots and pet robots. The most frequently cited barriers were mapped onto constructs within the domain "Intervention characteristics", while most facilitators were mapped onto the domain "Patients needs and resources". 
Table 4 Summary of barriers and facilitators

\begin{tabular}{|c|c|c|}
\hline CFIR construct & Barrier(s) & Facilitator(s) \\
\hline \multicolumn{3}{|c|}{ Domain 1. Innovation Characteristics } \\
\hline $\begin{array}{l}\text { 1.1 Relative } \\
\text { advantage }\end{array}$ & $\begin{array}{l}\text { - Relative cost as compared to other technology [70] } \\
\text { - Less audibility }[38,75,76]\end{array}$ & $\begin{array}{l}\text { - Sense of presence }[38,70,74-76] \\
\text { - Mobility aspect }[74] \text {. } \\
\text { - More conducive for people with dementia }[70,74] \\
\text { - Maintenance-free }[60,72] \\
\text { - Proactivity [56] } \\
\text { - Economic advantage }[59]\end{array}$ \\
\hline 1.2 Adaptability & $\begin{array}{l}\text { - Vocalisations [83] } \\
\text { - Functions [45] } \\
\text { - User interface or interaction }[41,46,53,63] \\
\text { - Physical inaccessibility }[41,47,59,68,74,77,78,81 \text {, } \\
\text { 84] }\end{array}$ & $\begin{array}{l}\text { - Physical accessibility }[41,74] \\
\text { - Customisability of interactivity or functions }[47,64]\end{array}$ \\
\hline 1.3 Complexity & $\begin{array}{l}\text { - Pre-programmed instructions }[39,46] \\
\text { - Complicated functions }[39,41,75-77,83,85,88] \\
\text { - Compose or program activities }[61] \\
\text { - Multimodal interaction features }[41,67,75,76]\end{array}$ & • Ease of use $[39-41,52,57,59-61,65,68,74,76,81,88]$ \\
\hline $\begin{array}{l}1.4 \text { Design quality } \\
\text { and packaging }\end{array}$ & $\begin{array}{l}\text { - Audio and speech issues }[39-41,43,46,47,53,63,71 \text {, } \\
74-76,81,84,86,88,89], \\
\text { - Hardware problems }[43,58,70] \\
\text { - Unreliable functions }[39,40,43,45,46,58,59,65,71 \text {, } \\
81,85,89,90] \text {, } \\
\text { - Unpredictable intentions } \\
{[39,40,43,51]} \\
\text { - Other technical difficulties }[43,47,54,61,77] \\
\text { - Physical attributes }[16,45,67,68,72,83] \\
\text { - Design }[71,72,81,83]\end{array}$ & $\begin{array}{l}\text { - Acceptable and/or pleasant appearance }[41,45,54,63,64,67,68 \text {, } \\
82,86,88] \\
\text { - Interactivity and proactivity }[40,41,57,58,77,84,85] \text {, } \\
\text { - Robustness }[44,57,89]\end{array}$ \\
\hline 1.5 Cost & $\begin{array}{l}\text { - High acquisition and maintenance cost }[44,57,69,72 \text {, } \\
77,83,88]\end{array}$ & \\
\hline
\end{tabular}

\section{Domain 2: Outer setting}

2.1 Patient needs and resources
- Unfamiliar with technology $[51,74,88]$

- Cognitive impairment [41, 48, 49, 53, 67, 74, 88]

- Independence in managing daily tasks $[60,77,88]$

- Limited usefulness of the robot $[40,41,45,51,57,65$, $83,87]$

- Doubts about sustained benefits $[57,86,88]$.

- Intrusiveness or privacy $[45,46,51,57,83,88]$

- Negative affect $[40,47,53,59,64,65,71,88]$

- Negative perceptions or stigma $[40,44,51,52,54,55$ $62,71,80,81,88]$

2.2 External policy/ • Align care work with national care policy [75, 76] incentives

\section{Domain 3: Inner Setting}

3.1 Compatibility • Institutional regulations: privacy, space and safety privacy $[38,75,84]$

- Confused/frightened residents [59]

- Background noises [41, 53, 75]

- Concern about misuse of technology [38, 75, 76]

- Lack of support from co-workers [61]

- Delineate professional boundary $[38,75,76]$

- Ethical concerns $[42,68,71,73]$

- Hygiene $[42,44,72,73]$

- Interfere with routine

- Physical environment [40]

3.2 Relative priority • Existing care work/processes took precedence [66, 68, 75]

- Workplace tension [68]

3.3 Leadership engagement

3.4 Available resources
- Support and familiarisation [47, 57, 79, 88]

- Emotional support [41, 52, 57, 58, 60, 82-85]

- Companionship [44, 45, 60, 77, 82, 83]

- Improvement to daily life $[40,58,63,81]$

- Entertainment $[41,45,50,63,64]$

- Reminiscence $[41,45,71]$

- Reminders $[54,58,64]$

- Phased introduction and training [46]

- Prolonged use $[46,47,51,70]$.
- Supported work of care professionals $[47,59,68,76,84]$

- Integration into care routine $[42,47,49,75,84]$

- Positioning of social robots [51, 60,65]

- Adaptation of physical environment $[40,41]$
- Poor network connectivity [38, 39, 55, 61, 68, 70, 74-
$76,81]$

- Lack of manpower, time or training $[42,66,68-70]$
- Leadership involvement and commitment [61]

- Improved network infrastructure [61]

- Time and support for care professionals [61]. 
Table 4 Summary of barriers and facilitators (Continued)

\begin{tabular}{|c|c|c|}
\hline CFIR construct & Barrier(s) & Facilitator(s) \\
\hline & - Computer incompatibility [74] & \\
\hline $\begin{array}{l}3.5 \text { Access to } \\
\text { knowledge and } \\
\text { information }\end{array}$ & - Access to support in rural areas [77] & $\begin{array}{l}\text { - Dedicated helpdesk within care facility [61] } \\
\text { - Individualised intervention instructions/manual [42, 43, 61] }\end{array}$ \\
\hline \multicolumn{3}{|c|}{ Domain 4: Characteristics of Individuals } \\
\hline $\begin{array}{l}4.1 \text { Knowledge and } \\
\text { beliefs }\end{array}$ & $\begin{array}{l}\text { - Initial ambivalence/negative attitudes }[42,47,59,66 \text {, } \\
68,72,74,81] \\
\text { - Fear of damaging robot }[59,77] \\
\text { - Privacy concern }[38,75,76] \\
\text { - Fear of job replacement }[47,59] \\
\text { - Negative perceptions, which stemmed from technical } \\
\text { challenges/ perceived lack of usefulness }[59,61,74 \text {, } \\
75]\end{array}$ & $\begin{array}{l}\text { - Evolved attitude after witnessing positive impacts on older adults/ } \\
\text { people with dementia }[42,44,47,49,56,66,68-70,72,74,75,80 \text {, } \\
81] \\
\text { - Understanding that robots cannot replace their jobs [47] } \\
\text { - Motivation to support robot interactions }[42,61,84] \\
\text { - Alignment to organisation visions [61] }\end{array}$ \\
\hline 4.2 Self-efficacy & - Unequipped to program and compose activities [61] & • Gain experience over time [61] \\
\hline \multicolumn{3}{|c|}{ Domain 5: Implementation Process } \\
\hline 5.1 Planning & - Assign robot with a clearly indicated role [84] & \\
\hline 5.2 Engaging & & $\begin{array}{l}\text { - Public exposure facilitated engagement and change in perceptions } \\
{[49,59,70]}\end{array}$ \\
\hline $\begin{array}{l}5.3 \text { Key } \\
\text { stakeholders }\end{array}$ & - Negative attitudes of care professionals [69] & $\begin{array}{l}\text { - Care professionals' enthusiasm [66] } \\
\text { - Active engagement with care professionals [84] } \\
\text { - Mediation of robot interactions }[43,47-50,67]\end{array}$ \\
\hline $\begin{array}{l}5.4 \text { External change } \\
\text { agents }\end{array}$ & • Lack of sustainability [47] & $\begin{array}{l}\text { - Support robot interactions }[40,41,49,74,76] \\
\text { - Provide technical support }[39,43,77]\end{array}$ \\
\hline
\end{tabular}

\section{Terminology}

Overall, less than a third of the articles included the term "implementation" in their title and/or abstracts. There appears to be no clear conceptual definition of the term "implementation". This could be attributed to different disciplinary research focus and/or disciplinespecific vocabulary, since included papers were derived from different academic fields: health and social sciences, engineering and computer science. In health and social science contexts, implementation refers to "the constellation of processes intended to get an intervention into use within an organisation" [32]. However, in computer science, it is used to describe the process of executing technical applications [92]. Given that social robotics is a transdisciplinary field, it is important for researchers to be aware of discipline-specific terms. Moving forward, a concept analysis should be done to understand interdisciplinary concepts used to describe implementation in relation to social robots. Terms in Proctor's taxonomy were identified in titles and/or abstracts of most included papers. This highlights the practicability of using the taxonomy to develop a sensitive search strategy to identify studies that investigated intervention implementation.

\section{Barriers}

Barriers to implementation were primarily related to the characteristics of social robots (i.e. "Intervention characteristics" domain), such as complexity, physical accessibility and cost.
In particular, technical failures were raised as issues in more than half of the included studies. It may be worth noting that most of these barriers were related to the use of socially assistive robots. This may be attributed to the range of functions available on such robots (as compared to telepresence or pet robots), which can proportionately increase the complexity of their operation. Although another possible explanation for barriers in this domain are that many of the social robots that were used were prototypes, it is also important to note that such issues were also raised in relation to the use of commercially available social robots such as Zora, Pepper and Giraff. Such challenges are not novel to social robots, as similar issues have been well-documented even amongst studies which used less novel or daily technology to conduct interventions [30, 93-95]. These issues had repercussions on other implementation domains, as they resulted in negative perceptions by multi-level key stakeholders, including older people and people with dementia, family members and care professionals. This finding is in alignment with findings by Rozental et al. [96], which found that such technical problems evoked negative psychological effect among users.

People with cognitive impairment required more support to use social robots, and those with less experience with technology had lower self-efficacy. This finding corresponded with existing research $[15,16,97,98]$. Next, the mismatch between the social robots' function and users' needs was also reported as an obstacle. Such barriers were primarily reported in studies which investigated the use of social robots for cognitively older adults 
who were living at home, suggesting that their needs and expectations of social robots differ from people with dementia or are living in care facilities, who may use technology differently. A recent scoping review by Abdi and colleagues [99] found that the needs of community-dwelling older adults ranged widely from mobility needs and interpersonal needs to selfmanagement needs. As such, they may require social robots to have more functionalities that are tailored to their needs $[83,100]$. In contrast, the needs of people with dementia and those in care setting differed. They included having stimulating day time activities and company [101]. Understanding of the needs of intended population is a therefore fundamental contextual consideration for implementing social robots.

Although one of the key bases for the development of social robots is to support and aid caregiving in individuals' homes and care settings [102], which is expected to be increasingly strained due to a rapidly aging population $[15,103]$, there is ironically a lack of studies which has investigated how social robots can be successfully integrated into care organisations (i.e. "Inner setting" domain). There were significantly more barriers than facilitators identified in this CFIR domain. These barriers, including incompatibility of the intervention to institutional regulations or work processes and the lack of time, manpower and training to support implementation efforts, corresponding with existing literature $[104,105]$. Therefore, dedicated resources should be allocated to supported the implementation of social robots, especially during the initial implementation phase [106] to allow care organisations and care professionals to familiarise and adapt to their use [107]. Next, even though organisational theories have highlighted the influence of other external factors on implementation such as external policies or incentives [108, 109], this was only reported in two studies. There is also a lack of studies that reported perspectives of other stakeholders, such as management staff and policy makers, which highlights research gaps in these areas. Finally, findings relating to the CFIR domain of "Implementation process" were scarce as there were few studies that undertook process evaluations.

\section{Facilitators}

Most of the identified facilitators correspond with the identified barriers. For instance, the characteristics of the social robots, such as their physical accessibility, ease of use, cost and technical robustness were identified as implementation facilitators. In addition, the match between social robots' functions and users' needs and their compatibility with work processes within care organisations were seen as enablers. We also found that despite initial ambivalence or scepticism, older adults and people with dementia developed positive perceptions after using social robots with functions that matches their needs or expectations. Similarly, when family members and care professionals experienced the positive impacts of the technology and developed a renewed understanding that they cannot replace their jobs, positive attitudes were reported. This confirms current research findings that direct experiences with a technology can elicit attitude change when the interactions evoke cognitive-affective discrepancies from baseline beliefs $[110,111]$. These positive perceptions had implications on other implementation domains. In the CFIR domain of "Implementation process", care professionals and family members who had had positive attitudes were more enthusiastic in supporting and facilitating robot interactions. The mediation of robot interactions by these stakeholders also helped to reconcile the limitations of the intervention characteristics, such as technical issues and the complexity of use. These facilitators also highlight the importance of avoiding evaluating implementation determinants in silos, and instead consider the interplay of multi-level contextual factors that influence implementation $[112,113]$.

\section{Future research and practical implications}

Overall, more barriers than facilitators were identified. Data from this review could only be mapped onto 18 out of 39 constructs in the CFIR. Data were mostly coded to the CFIR domain of "intervention characteristics", and there is significantly less research emphasis on other CFIR domains. This is also exemplified through the lack of data that could be mapped onto 21 other CFIR constructs. This indicates that existing research have been focused on the internal validity of the intervention, and that future research focus must be directed towards identifying other contextual factors that can influence the external validity of social robots in real-world practice. Very few of the included studies have undertaken process evaluations, and none have used an implementation framework to ensure a systematic approach to consider all factors that can affect implementation. Given the complexity of implementing social robots, process evaluations can provide valuable insights that may explain why the intervention has (or has not) been implemented as intended in real-world practice [114], and how different contextual factors may have influenced overall intervention outcomes [115]. Future research should also consider applying an appropriate theoretical framework to guide a thorough investigation of implementation determinants, which can then enable corresponding strategies to be identified and tested in realworld practice. Waltz and colleagues [116] developed a tool for mapping barriers identified on each CFIR domain to the Expert Recommendations for Implementing Change (ERIC), which contains a comprehensive 
collection of implementation strategies [117]. For instance, to address a barrier relating to "compatibility", one recommended strategy listed in CFIR-ERIC mapping tool is to conduct local consensus discussions, where different key stakeholders should engage in active discussions about whether social robots are appropriate to address needs within their context. Finally, aside from focusing on barriers, it is also pivotal to leverage on facilitators to guide the successful implementation of social robots in the realworld setting.

\section{Strengths and limitations}

There are a number of strengths underpinning this work. First, the methodological framework that was used was transparent and rigorous. We searched multiple databases, including grey literature and engineering databases. The application of an implementation science framework (i.e., the CFIR) enabled results to be presented in a comprehensive and systematic manner. Nevertheless, there are limitations of this review. In our review protocol, we reported a plan to extract terms used to describe implementation from the full text of included articles. However, due to the large number of articles that were included in this review, we had to deviate from the protocol to only chart terms that were included in the title and/or abstract of included papers. Articles that were published in other languages were not included in this review. Hence, relevant studies might be missed. In addition, the review aggregated barriers and facilitators related to the implementation of social robots in participants' home and long-term care settings, and thus the findings mainly apply to these settings. Several different social robots (i.e., interventions) were included in this review. The heterogeneity of the interventions and study settings could be a fundamental limitation, as these variable factors can affect implementation differently. Nevertheless, implementation barriers and facilitators that were identified in this study revolved around similar themes.

\section{Conclusion}

This review has identified and synthesised terms used to describe implementation in relation to social robots, and the breadth of evidence on the barriers and facilitators to the implementation of social robots for older adults and people with dementia. There is a lack of clear conceptual clarity regarding the term "implementation". A concept analysis may be warranted to explore this topic in depth. Although social robots show promise for improving the psychosocial health of older adults and people with dementia, there has been little attention paid to their implementation in the real-world setting. Most existing research were focused on evaluating the characteristics of social robots, and there has been significantly less research which investigated other multi-level contextual factors, such as organisational or wider contextual factors, that can influence their implementation in real-world practice. Further research in these domains, using an implementation framework, is necessitated.

Abbreviation

CFIR: Consolidated Framework of Implementation Research

\section{Supplementary Information}

The online version contains supplementary material available at https://doi. org/10.1186/s12877-021-02277-9.

Additional file 1.

Additional file 2.

Additional file 3.

Additional file 4

\section{Acknowledgements}

We would like to thank Ms. Rosie Dunnes for her contribution to the development of our literature search strategy.

\section{Authors' contributions}

WK conceptualised the review approach, developed the review questions and review design with advice from ET and DC, and conducted the literature search. WK, KB and SF undertook the screening, study selection and data charting process. WK undertook screening of the reference lists and backward citation tracing. Data synthesis was led by WK with consultation with ET. WK initiated the draft of the manuscript. ET and DC had meaningful contributions to its drafting and editing. All authors read and approved the final manuscript.

\section{Funding}

The research presented in this paper was carried out as part of the Marie Curie Innovative Training Network (ITN) action, H2020-MSCA-ITN-2018, under grant agreement number 813196.

Availability of data and materials

Supporting data and materials used in this paper can be accessed online through various public databases. The datasets used and/or analysed during the current study are available from the corresponding author on reasonable request.

\section{Declarations}

Ethics approval and consent to participate

Not applicable.

Consent for publication

Not applicable.

\section{Competing interests}

The authors declare that they have no competing interests.

Author details

${ }^{1}$ National University of Ireland Galway, H91 E3YV Galway, Ireland. ${ }^{2}$ German Center for Neurodegenerative Diseases Witten, Stockumer Str. 12, 58452 Witten, Germany. ${ }^{3}$ University of Limerick, V94 T9PX Limerick, Ireland.

Received: 24 February 2021 Accepted: 10 May 2021

Published online: 09 June 2021

\section{References}

1. World Health Organization. Aging and Health 2018 [updated 5 Feb 2018]. Available from https://www.who.int/news-room/fact-sheets/detail/ageing-a nd-health. Accessed 20 Jan 2021. 
2. World Health Organization. Dementia 2020 [updated 21 September 2020]. Available from: https:/www.who.int/news-room/fact-sheets/detail/ dementia. Accessed 20 Jan 2021.

3. von Strauss E, Viitanen M, De Ronchi D, Winblad B, Fratiglioni L. Aging and the occurrence of dementia: findings from a population-based cohort with a large sample of nonagenarians. Arch Neurol. 1999;56(5):587-92. https:// doi.org/10.1001/archneur.56.5.587.

4. Werth JL Jr, Gordon JR, Johnson RR Jr. Psychosocial issues near the end of life. Aging Ment Health. 2002;6(4):402-12. https://doi.org/10.1080/13 60786021000007027.

5. Brady M. Pre-hospital psychosocial care: changing attitudes. J Paramed Pract. 2012;4(9):516-25. https://doi.org/10.12968/jpar.2012.4.9.516.

6. Cristea M, Noja GG, Stefea P, Sala AL. The impact of population aging and public health support on EU labor markets. Int J Environ Res Public Health. 2020;17(4):1439. https://doi.org/10.3390/ijerph17041439.

7. Dautenhahn K. Socially intelligent robots: dimensions of human-robot interaction. Philos Trans R Soc B: Biol Sci. 2007;362(1480):679-704. https:// doi.org/10.1098/rstb.2006.2004.

8. Fong T, Nourbakhsh I, Dautenhahn K. A survey of socially interactive robots. Robot Auton Syst. 2003;42(3-4):143-66. https://doi.org/10.1016/S09218890(02)00372-X.

9. Miklósi Á, Gácsi M. On the utilization of social animals as a model for social robotics. Front Psychol. 2012;3:75.

10. Abdi J, Al-Hindawi A, Ng T, Vizcaychipi MP. Scoping review on the use of socially assistive robot technology in elderly care. BMJ Open. 2018;8(2): e018815. https://doi.org/10.1136/bmjopen-2017-018815.

11. Feil-Seifer D, Mataric MJ. Defining socially assistive robotics. 19th International Conference on Rehabilitation Robotics (ICORR), 2005. Chicago; 2005. p. 465-8.

12. Leng M, Liu P, Zhang P, Hu M, Zhou H, Li G, et al. Pet robot intervention for people with dementia: a systematic review and meta-analysis of randomized controlled trials. Psychiatry Res. 2019;271:516-25. https://doi. org/10.1016/j.psychres.2018.12.032.

13. Abbott R, Orr N, McGill P, Whear R, Bethel A, Garside R, et al. How do "robopets" impact the health and well-being of residents in care homes? A systematic review of qualitative and quantitative evidence. Int J Older People Nursing. 2019;14(3):e12239. https://doi.org/10.1111/opn.12239.

14. Stahl C, Anastasiou D, Latour T. Social Telepresence Robots: The role of gesture for collaboration over a distance. In: Proceedings of the 11th PErvasive Technologies Related to Assistive Environments Conference; 2018.

15. Hung L, Liu C, Woldum E, Au-Yeung A, Berndt A, Wallsworth C, et al. The benefits of and barriers to using a social robot PARO in care settings: a scoping review. BMC Geriatr. 2019;19(1):232. https://doi.org/10.1186/s12877-019-1244-6.

16. Pu L, Moyle W, Jones $\mathrm{C}$, Todorovic M. The effectiveness of social robots for older adults: a systematic review and meta-analysis of randomized controlled studies. The Gerontologist. 2019;59(1):e37-51. https://doi.org/10.1 093/geront/gny046.

17. Chen SC, Jones C, Moyle W. Social robots for depression in older adults: a systematic review. J Nurs Scholarsh. 2018;50(6):612-22. https://doi.org/1 $0.1111 /$ jnu. 12423.

18. Meiland F, Innes A, Mountain G, Robinson L, van der Roest H, García-Casal $J A$, et al. Technologies to support community-dwelling persons with dementia: a position paper on issues regarding development, usability, effectiveness and cost-effectiveness, deployment, and ethics. JMIR Rehabil Assist Technol. 2017:4(1):e1. https://doi.org/10.2196/rehab.6376.

19. Moniz-Cook E, Vernooij-Dassen M. Raising the standard of applied dementia care research: addressing the implementation error. Aging Mental Health. 2014;18(7):809-14.

20. Klein B, Gaedt L, Cook G. Emotional robots: Principles and experiences with Paro in Denmark, Germany, and the UK. GeroPsych: The Journal of Gerontopsychology and Geriatric Psychiatry. 2013;26(2):89-99. https://doi. org/10.1024/1662-9647/a000085

21. The Alzheimer Society of Ireland. A first for Irish Dementia Day Care as The Alzheimer Society of Ireland Introduces Paro the Therapeutic Robot to Persons with Dementia; 2018. Available from: https://alzheimer.ie/creatingchange/awareness-raising/dementia-in-the-media/. Accessed 21 Jan 2021.

22. Bauer MS, Damschroder L, Hagedorn H, Smith J, Kilbourne AM. An introduction to implementation science for the non-specialist. BMC Psychol. 2015;3(1):32. https://doi.org/10.1186/s40359-015-0089-9.

23. Glasgow RE, Lichtenstein E, Marcus AC. Why don't we see more translation of health promotion research to practice? Rethinking the efficacy-to- effectiveness transition. Am J Public Health. 2003;93(8):1261-7. https://doi. org/10.2105/AJPH.93.8.1261.

24. Tunis SR, Stryer DB, Clancy CM. Practical clinical trials: increasing the value of clinical research for decision making in clinical and health policy. JAMA. 2003;290(12):1624-32. https://doi.org/10.1001/jama.290.12.1624.

25. Proctor EK, Landsverk J, Aarons G, Chambers D, Glisson C, Mittman B. Implementation research in mental health services: an emerging science with conceptual, methodological, and training challenges. Adm Policy Mental Health Mental Health Serv Res. 2009;36(1):24-34. https://doi.org/10.1 007/s10488-008-0197-4.

26. Landes SJ, McBain SA, Curran GM. Reprint of: an introduction to effectiveness-implementation hybrid designs. Psychiatry Res. 2020;283: 112630. https://doi.org/10.1016/j.psychres.2019.112630.

27. Curran GM, Bauer M, Mittman B, Pyne JM, Stetler C. Effectivenessimplementation hybrid designs: combining elements of clinical effectiveness and implementation research to enhance public health impact. Med Care. 2012;50(3):217-26. https://doi.org/10.1097/MLR.0b013e31 82408812.

28. Papadopoulos I, Koulouglioti C, Lazzarino R, Ali S. Enablers and barriers to the implementation of socially assistive humanoid robots in health and social care: a systematic review. BMJ Open. 2020;10(1):e033096. https://doi. org/10.1136/bmjopen-2019-033096.

29. Colquhoun H, Leeman J, Michie S, Lokker C, Bragge P, Hempel S, et al. Towards a common terminology: a simplified framework of interventions to promote and integrate evidence into health practices, systems, and policies. Implement Sci. 2014;9(1):1-6.

30. Christie HL, Bartels SL, Boots LM, Tange HJ, Verhey FR, de Vugt ME. A systematic review on the implementation of eHealth interventions for informal caregivers of people with dementia. Internet Interv. 2018;13:51-9. https://doi.org/10.1016/j.invent.2018.07.002.

31. Arksey H, O'Malley L. Scoping studies: towards a methodological framework. Int J Soc Res Methodol. 2005;8(1):19-32. https://doi.org/10.1080/1364557032 000119616.

32. Damschroder $L$, Aron DC, Keith RE, Kirsh SR, Alexander JA, Lowery JC. Fostering implementation of health services research findings into practice: a consolidated framework for advancing implementation science. Implement Sci. 2009;4(1):1-15.

33. Levac D, Colquhoun H, O'Brien KK. Scoping studies: advancing the methodology. Implement Sci. 2010;5(1):1-9.

34. Tricco AC, Lillie E, Zarin W, O'Brien KK, Colquhoun H, Levac D, et al. PRISMA extension for scoping reviews (PRISMA-ScR): checklist and explanation. Ann Intern Med. 2018;169(7):467-73. https://doi.org/10.7326/M18-0850.

35. Koh WQ, Felding SA, Toomey E, Casey D. Barriers and facilitators to the implementation of social robots for older adults and people with dementia: a scoping review protocol. Syst Rev. 2021;10(1):1-6.

36. Proctor E, Silmere H, Raghavan R, Hovmand P, Aarons G, Bunger A, et al. Outcomes for implementation research: conceptual distinctions, measurement challenges, and research agenda. Adm Policy Mental Health Mental Health Serv Res. 2011;38(2):65-76. https://doi.org/10.1007/s10488-01 0-0319-7.

37. Horsley T, Dingwall O, Sampson M. Checking reference lists to find additional studies for systematic reviews. Cochrane Database Syst Rev. 2011(8). https://doi.org/10.1002/14651858.MR000026.pub2.

38. Aaltonen I, Niemelä M, Tammela A. Please call me? Calling practices with telepresence robots for the elderly. In: Proceedings of the Companion of the 2017 ACM/IEEE International Conference on Human-Robot Interaction; 2017.

39. Bajones $M$, Fischinger $D$, Weiss $A$, Wolf $D$, Vincze $M$, de la Puente $P$, et al. Hobbit: providing fall detection and prevention for the elderly in the real world. J Robot. 2018;2018:1-20. https://doi.org/10.1155/2018/1754657.

40. Bajones $M$, Fischinger $D$, Weiss $A$, Puente PDL, Wolf $D$, Vincze $M$, et al Results of field trials with a Mobile service robot for older adults in 16 private households. ACM Trans Hum-Robot Interact. 2019;9(2):1-27.

41. Barrett E, Burke M, Whelan S, Santorelli A, Oliveira BL, Cavallo F, et al. Evaluation of a companion robot for individuals with dementia: quantitative findings of the MARIO project in an Irish residential care setting. J Gerontol Nurs. 2019:45(7):36-45. https://doi.org/10.3928/00989134-20190531-01.

42. Bemelmans R, Gelderblom GJ, Jonker P, de Witte L. How to use robot interventions in intramural psychogeriatric care; a feasibility study. Appl Nurs Res. 2016;30:154-7. https://doi.org/10.1016/j.apnr.2015.07.003.

43. Blond L. Studying robots outside the lab: HRI as ethnography. J Behav Robot. 2019;10(1):117-27. https://doi.org/10.1515/pjbr-2019-0007. 
44. Bradwell HL, Winnington R, Thill S, Jones RB. Longitudinal diary data: six months real-world implementation of affordable companion robots for older people in supported living. In: Companion of the 2020 ACM/IEEE International Conference on Human-Robot Interaction; 2020.

45. Broadbent E, Peri K, Kerse N, Jayawardena C, Kuo I, Datta C, MacDonald B. Robots in older people's homes to improve medication adherence and quality of life: a randomised cross-over trial. International conference on social robotics. Sydney: Springer, Cham; 2014. p. 64-73.

46. Caleb-Solly P, Dogramadzi S, Huijnen CA, Heuvel HJTIS. Exploiting ability for human adaptation to facilitate improved human-robot interaction and acceptance. Inf Soc. 2018;34(3):153-65.

47. Carros F, Meurer J, Löffler D, Unbehaun D, Matthies S, Koch I, et al. Exploring human-robot interaction with the elderly: results from a ten-week case study in a care home. In: Proceedings of the $2020 \mathrm{CHI}$ Conference on Human Factors in Computing Systems; 2020.

48. Chang WL, Šabanović S, Huber L. Situated analysis of interactions between cognitively impaired older adults and the therapeutic robot PARO. International Conference on Social Robotics. Bristol, UK: Springer, Cham; 2013, p. 371-80.

49. Chang WL, Sabanovic S. Interaction expands function: Social shaping of the therapeutic robot PARO in a nursing home. HRI '15: Proceedings of the Tenth Annual ACM/IEEE International Conference on Human-Robot Interaction. Portland Oregon; 2015. p. 343-50.

50. Cruz-Sandoval D, Favela J, Sandoval EB. Strategies to facilitate the acceptance of a social robot by people with dementia. In: Companion of the 2018 ACM/ IEEE International Conference on Human-Robot Interaction; 2018.

51. De Graaf MM, Allouch SB, Klamer TJC. Sharing a life with Harvey: exploring the acceptance of and relationship-building with a social robot. Comput Hum Behav. 2015;43:1-14.

52. Demange M, Lenoir H, Pino M, Cantegreil-Kallen I, Rigaud AS, CristanchoLacroix V. Improving well-being in patients with major neurodegenerative disorders: differential efficacy of brief social robot-based intervention for 3 neuropsychiatric profiles. Clin Interv Aging. 2018;13:1303-11. https://doi. org/10.2147/CIA.S152561.

53. D'Onofrio G, Sancarlo D, Raciti M, Burke M, Teare A, Kovacic T, et al. MARIO project: validation and evidence of service robots for older people with dementia. J Alzheimers Dis. 2019;68(4):1587-601. https://doi.org/10.3233/JAD-181165.

54. Fattal C, Cossin I, Pain F, Haize E, Marissael C, Schmutz S, et al. Perspectives on usability and accessibility of an autonomous humanoid robot living with elderly people. Disabil Rehabil: Assist Technol. 2020:1-13. https://doi.org/1 0.1080/17483107.2020.1786732.

55. Fiorini L, Mancioppi G, Becchimanzi C, Sorrentino A, Pistolesi M, Tosi F, Cavallo F. Multidimensional evaluation of telepresence robot: results from a field trial. 29th IEEE International Conference on Robot and Human Interactive Communication (RO-MAN). Naples; 2020. p. 1211-6.

56. Gross HM, Schroeter C, Müller S, Volkhardt M, Einhorn E, Bley A, Langner T, Merten M, Huijnen C, van den Heuvel, $H$ van Berlo A. Further progress towards a home robot companion for people with mild cognitive impairment. 2012 IEEE International Conference on Systems, Man, and Cybernetics (SMC). Seoul; 2012. p. 637-44.

57. Gross HM, Mueller S, Schroeter C, Volkhardt M, Scheidig A, Debes K, Richter K, Doering N. Robot companion for domestic health assistance: Implementation, test and case study under everyday conditions in private apartments. IEEE/RSJ International Conference on Intelligent Robots and Systems (IROS); Hamburg; 2015. p. 5992-9.

58. Gross HM, Scheidig A, Müller S, Schütz B, Fricke C, Meyer S. Living with a mobile companion robot in your own apartment-final implementation and results of a 20-weeks field study with 20 seniors. 2019 International Conference on Robotics and Automation (ICRA). Montreal: IEEE; 2019. p. 2253-9.

59. Hebesberger D, Koertner T, Gisinger C, Pripfl J. A long-term autonomous robot at a care hospital: a mixed methods study on social acceptance and experiences of staff and older adults. Int J Soc Robot. 2017;9(3):417-29. https://doi.org/10.1007/s12369-016-0391-6.

60. Hudson J, Ungar R, Albright L, Tkatch R, Schaeffer J, Wicker ER. Robotic pet use among community-dwelling older adults. J Gerontol: Series B. 2020; 75(9):2018-28. https://doi.org/10.1093/geronb/gbaa119.

61. Huisman C, Kort H. Two-year use of care robot Zora in Dutch nursing homes: an evaluation study. Healthcare. 2019;7(1):31. https://doi.org/10.33 90/healthcare7010031.

62. Kelly PA, Cox LA, Petersen SF, Gilder RE, Blann A, Autrey AE, et al. The effect of PARO robotic seals for hospitalized patients with dementia: a feasibility study. Geriatr Nurs. 2020;42(1):37-45. https://doi.org/10.1016/j.gerinurse.202 0.11.003.

63. Khosla R, Nguyen K, Chu M-T. Human robot engagement and acceptability in residential aged care. Int J Hum-Comput Interact. 2017;33(6):510-22. https://doi.org/10.1080/10447318.2016.1275435.

64. Khosla R, Chu MT, Khaksar SM, Nguyen K, Nishida T. Engagement and experience of older people with socially assistive robots in home care. Assist Technoly. 20214;33(2):57-71.

65. Klamer T, Allouch SB. Acceptance and use of a social robot by elderly users in a domestic environment. 2010 4th International Conference on Pervasive Computing Technologies for Healthcare. Munich; 2010. p. 1-8.

66. Kolstad M, Yamaguchi N, Babic A, Nishihara Y. Integrating socially assistive robots into Japanese nursing care. Stud Health Technol Inf. 2020;270:13234. https://doi.org/10.3233/SHTI200423.

67. Kouroupetroglou C, Casey D, Raciti M, Barrett E, D'Onofrio G, Ricciardi F, et al. Interacting with dementia: the MARIO approach. Stud Health Technol Inform. 2017;242:38-47.

68. Melkas H, Hennala L, Pekkarinen S, Kyrki V. Impacts of robot implementation on care personnel and clients in elderly-care institutions. Int J Med Inform. 2020;134:104041. https://doi.org/10.1016/j.jjmedinf.2019.104041.

69. Moyle W, Jones C, Cooke M, O'Dwyer S, Sung B, Drummond S. Social robots helping people with dementia: assessing efficacy of social robots in the nursing home environment. In: 2013 6th International Conference on Human System Interactions (HSI): IEEE; 2013.

70. Moyle W, Jones C, Cooke M, O'Dwyer S, Sung B, Drummond S. Connecting the person with dementia and family: a feasibility study of a telepresence robot. BMC Geriatr. 2014;14(1):7. https://doi.org/10.11 86/1471-2318-14-7.

71. Moyle W, Jones C, Sung B, Bramble M, O'Dwyer S, Blumenstein M, et al. What effect does an animal robot called CuDDler have on the engagement and emotional response of older people with dementia? A pilot feasibility study. Int J Soc Robot. 2016;8(1):145-56. https://doi.org/10.1007/s12369-015-0326-7.

72. Moyle W, Bramble M, Jones CJ, Murfield JE. "She had a smile on her face as wide as the great Australian bite": a qualitative examination of family perceptions of a therapeutic robot and a plush toy. The Gerontologist. 2019; 59(1):177-85. https://doi.org/10.1093/geront/gnx180.

73. Moyle W, Jones C, Murfield J, Thalib L, Beattie E, Shum D, et al. Using a therapeutic companion robot for dementia symptoms in long-term care: reflections from a cluster-RCT. Aging Ment Health. 2019;23(3):329-36. https://doi.org/10.1080/13607863.2017.1421617.

74. Moyle W, Jones C, Sung B. Telepresence robots: encouraging interactive communication between family carers and people with dementia. Aust J Ageing. 2019;39(1):e127-e33.

75. Niemelä M, van Aerschot L, Tammela A, Aaltonen I. A telepresence robot in residential care: Family increasingly present, personnel worried about privacy. In: Kheddar A, Yoshida E, Ge S, Suzuki K, Cabibihan J, Eysel F, He H. Social robotics. International Conference on Social Robotics; Tsukuba: Springer, Cham; 2017. p. 85-94.

76. Niemelä M, Van Aerschot L, Tammela A, Aaltonen I, Lammi H. Towards ethical guidelines of using telepresence robots in residential care. Int J Soc Robot. 2019:1-9. https://doi.org/10.1007/s12369-019-00529-8.

77. Orejana JR, MacDonald BA, Ahn HS, Peri K, Broadbent E. Healthcare robots in homes of rural older adults. International Conference on Social Robotics; Paris: Springer, Cham; 2015. p. 512-21.

78. Peri K, Kerse N, Broadbent E, Jayawardena C, Kuo T, Datta C, et al. Lounging with robots-social spaces of residents in care: a comparison trial. Australas J Ageing. 2016;35(1):E1-6. https://doi.org/10.1111/ajag.12201.

79. Piasek J, Wieczorowska-Tobis K. Acceptance and long-term use of a social robot by elderly users in a domestic environment. 11th International Conference on Human System Interaction (HSI). Gdańsk; 2018. p. 478-82.

80. Pike J, Picking R, Cunningham S. Robot companion cats for people at home with dementia: A qualitative case study on companotics, Dementia. 2020. https://doi.org/10.1177/1471301220932780.

81. Portugal D, Alvito P, Christodoulou E, Samaras G, Dias J. A study on the deployment of a service robot in an elderly care center. Int J Soc Robot. 2019;11(2):317-41. https://doi.org/10.1007/s12369-018-0492-5.

82. Pu L, Moyle W, Jones C. How people with dementia perceive a therapeutic robot called PARO in relation to their pain and mood: a qualitative study. J Clin Nurs. 2020;29(3-4):437-46. https://doi.org/10.1111/jocn.15104.

83. Randall N, Bennett CC, Šabanović S, Nagata S, Eldridge L, Collins S, et al. More than just friends: in-home use and design recommendations for 
sensing socially assistive robots (SARs) by older adults with depression. $J$ Behav Robot. 2019;10(1):237-55. https://doi.org/10.1515/pjbr-2019-0020.

84. Sabelli AM, Kanda T, Hagita N. A conversational robot in an elderly care center: an ethnographic study. 6th ACM/IEEE international conference on human-robot interaction (HRI). Lausanne; 2011. p. 37-44

85. Schroeter C, Mueller S, Volkhardt M, Einhorn E, Huijnen C, van den Heuvel $H$, van Berlo A, Bley A, Gross HM. Realization and user evaluation of a companion robot for people with mild cognitive impairments. 2013 IEEE International Conference on robotics and automation. Karlsruhe; 2013. p. 1153-9.

86. Torta E, Werner F, Johnson DO, Juola JF, Cuijpers RH, Bazzani M, et al. Evaluation of a small socially-assistive humanoid robot in intelligent homes for the care of the elderly. J Intell Robot Syst. 2014;76(1):57-71. https://doi. org/10.1007/s10846-013-0019-0

87. Van Maris A, Zook N, Caleb-Solly P, Studley M, Winfield A, Dogramadzi S. Designing ethical social robots - a longitudinal field study with older adults. Front Robot Al. 2020;7:1. https://doi.org/10.3389/frobt.2020.00001.

88. Wu Y-h, Wrobel J, Cornuet M, Kerhervé H, Damnée S, Rigaud A-S. Acceptance of an assistive robot in older adults: a mixed-method study of human-robot interaction over a 1-month period in the living lab setting Clin Interv Aging. 2014;9:801.

89. Zsiga K, Tóth A, Pilissy T, Péter O, Dénes Z, Fazekas G. Evaluation of a companion robot based on field tests with single older adults in their homes. Assist Technol. 2018;30(5):259-66. https://doi.org/10.1080/1040043 5.2017.1322158

90. D'Onofrio G, Sancarlo D, Raciti M, Reforgiato D, Mangiacotti A, Russo A, Ricciardi F, Vitanza A, Cantucci F, Presutti V, Messervey T. MARIO Project: Experimentation in the Hospital Setting. In: Casiddu N, Porfirione C, Monteriu A, Cavallo F. Ambient Assisted Living. Italian Forum of Ambient Assisted Living. Genova: Springer, Cham; 2017. 289-303.

91. Bradwell HL, Edwards KJ, Winnington R, Thill S, Jones RB. Companion robots for older people: importance of user-centred design demonstrated through observations and focus groups comparing preferences of older people and roboticists in south West England. BMJ Open. 2019;9(9):e032468. https://doi. org/10.1136/bmjopen-2019-032468.

92. Stanford Encylopedia of Philosophy. The Philosophy of Computer Science 2013 [updated 19 January, 2021]. Available from: https://plato.stanford.edu/ entries/computer-science/\#lmpl. Accessed 20 Apr 2021.

93. Airola E, Rasi P, Outila M. Older people as users and non-users of a video conferencing service for promoting social connectedness and well-being-a case study from Finnish Lapland. Educ Gerontol. 2020;46(5):258-69. https:// doi.org/10.1080/03601277.2020.1743008.

94. Baker S, Warburton J, Hodgkin S, Pascal J. The supportive network: rural disadvantaged older people and ICT. Ageing Soc. 2016;37(6):1291-309.

95. Chi N-C, Sparks O, Lin S-Y, Lazar A, Thompson HJ, Demiris G. Pilot testing a digital pet avatar for older adults. Geriatr Nurs. 2017;38(6):542-7. https://doi. org/10.1016/j.gerinurse.2017.04.002.

96. Rozental A, Boettcher J, Andersson G, Schmidt B, Carlbring P. Negative effects of internet interventions: a qualitative content analysis of patients' experiences with treatments delivered online. Cogn Behav Ther. 2015;44(3): 223-36. https://doi.org/10.1080/16506073.2015.1008033.

97. Heerink M. Exploring the influence of age, gender, education and computer experience on robot acceptance by older adults. 2011 6th ACM/IEEE International Conference on Human-Robot Interaction (HRI). Lausanne; 2011. p. 147-8.

98. Flandorfer P. Population ageing and socially assistive robots for elderly persons: the importance of sociodemographic factors for user acceptance. Int J Popul Res. 2012;2012:1-13. https://doi.org/10.1155/2012/829835.

99. Abdi S, Spann A, Borilovic J, de Witte L, Hawley M. Understanding the care and support needs of older people: a scoping review and categorisation using the $\mathrm{WHO}$ international classification of functioning, disability and health framework (ICF). BMC Geriatr. 2019;19(1):195. https://doi.org/10.1186/ s12877-019-1189-9.

100. Park Y-H, Chang HK, Lee MH, Lee SH. Community-dwelling older adults' needs and acceptance regarding the use of robot technology to assist with daily living performance. BMC Geriatr. 2019;19(1):208. https://doi.org/10.11 86/s12877-019-1227-7.

101. Hancock GA, Woods B, Challis D, Orrell M. The needs of older people with dementia in residential care. J Int J Geriatr Psychiatry. 2006;21(1):43-9. https://doi.org/10.1002/gps.1421.
102. Baer M, Tilliette M-A, Jeleff A, Ozguler A, Loeb T. Assisting older people: from robots to drones. Gerontechnology. 2014;13(1):57-8.

103. Pedersen I, Reid S, Aspevig K. Developing social robots for aging populations: a literature review of recent academic sources. Sociol Compass. 2018;12(6):e12585. https://doi.org/10.1111/soc4.12585.

104. Kormelinck CMG, Janus SI, Smalbrugge M, Gerritsen DL, Zuidema SU. Systematic review on barriers and facilitators of complex interventions for residents with dementia in long-term care. Int Psychogeriatr. 2020:1-17. https://doi.org/10.1017/S1041610220000034.

105. Geerligs L, Rankin NM, Shepherd HL, Butow P. Hospital-based interventions: a systematic review of staff-reported barriers and facilitators to implementation processes. Implement Sci. 2018;13(1):36. https://doi.org/1 0.1186/s13012-018-0726-9.

106. Chua KSG, Kuah CWK. Innovating with rehabilitation technology in the real world: promises, potentials, and perspectives. Am J Phys Med Rehabil. 2017; 96(10 Suppl 1):S150-6. https://doi.org/10.1097/PHM.0000000000000799.

107. Turchetti G, Vitiello N, Romiti S, Geisler E, Micera S. Why effectiveness of robot-mediated neurorehabilitation does not necessarily influence its adoption. IEEE Rev Biomed Eng. 2014;7:143-53. https://doi.org/10.1109/ RBME.2014.2300234.

108. Cabana MD, Rand CS, Powe NR, Wu AW, Wilson MH, Abboud P-AC, et al. Why don't physicians follow clinical practice guidelines?: a framework for improvement. JAMA. 1999;282(15):1458-65. https://doi.org/10.1001/jama.2 82.15.1458.

109. Hamilton AB, Mittman BS, Eccles AM, Hutchinson CS, Wyatt GE. Conceptualizing and measuring external context in implementation science: studying the impacts of regulatory, fiscal, technological and social change. Implement Sci. 2015;10(1):1-2. https://doi.org/10.1186/1748-5908-10-S1-A72.

110. Bhattacherjee A, Premkumar G. Understanding changes in belief and attitude toward information technology usage: a theoretical model and longitudinal test. MIS Q. 2004;28(2):229-54. https://doi.org/10.2307/25148634.

111. Damholdt MF, Nørskov M, Yamazaki R, Hakli R, Hansen CV, Vestergaard C, et al. Attitudinal change in elderly citizens toward social robots: the role of personality traits and beliefs about robot functionality. Front Psychol. 2015;6:1701.

112. Urquhart R, Porter GA, Sargeant J, Jackson L, Grunfeld E. Multi-level factors influence the implementation and use of complex innovations in cancer care: a multiple case study of synoptic reporting. Implement Sci. 2014;9(1): 121. https://doi.org/10.1186/s13012-014-0121-0.

113. Ross J, Stevenson F, Lau R, Murray E. Factors that influence the implementation of e-health: a systematic review of systematic reviews (an update). Implement Sci. 2016;11(1):146. https://doi.org/10.1186/s13012-0160510-7.

114. Moore GF, Audrey S, Barker M, Bond L, Bonell C, Hardeman W, et al. Process evaluation of complex interventions: Medical Research Council guidance. BMJ. 2015;350(mar19 6). https://doi.org/10.1136/bmj.h1258.

115. Oakley A, Strange V, Bonell C, Allen E, Stephenson J. Process evaluation in randomised controlled trials of complex interventions. BMJ. 2006;332(7538): 413-6. https://doi.org/10.1136/bmj.332.7538.413.

116. Waltz TJ, Powell BJ, Fernández ME, Abadie B, Damschroder LJ. Choosing implementation strategies to address contextual barriers: diversity in recommendations and future directions. Implement Sci. 2019;14(1):1-15.

117. Powell BJ, Waltz TJ, Chinman MJ, Damschroder LJ, Smith JL, Matthieu MM, et al. A refined compilation of implementation strategies: results from the expert recommendations for implementing change (ERIC) project. Implement Sci. 2015;10(1):1-14.

\section{Publisher's Note}

Springer Nature remains neutral with regard to jurisdictional claims in published maps and institutional affiliations. 